\title{
An ethanolic extract of the aerial part of Siegesbeckia orientalis L. inhibits the production of inflammatory mediators regulated by AP-1, NF-KB and IRF3 in LPS-stimulated RAW 264.7 cells
}

\author{
Hui Guo ${ }^{1}$, Yi Zhang ${ }^{1}$, Brian Chiyan Cheng ${ }^{1}$, Xiuqiong Fu ${ }^{1}$, Peili Zhu ${ }^{1}$, Jiali Chen ${ }^{1}$, \\ Yuencheung Chan ${ }^{1}$, Chengle Yin ${ }^{1}$, Yaping Wang ${ }^{1}$, Muhammadjahangir Hossen ${ }^{1}$, Aftab Amin ${ }^{1}$, \\ Anfernee Kaiwing Tse ${ }^{1}$, Zhi-ling $\mathbf{Y u}^{1,2, *}$ \\ ${ }^{1}$ Centre for Cancer and Inflammation Research, School of Chinese Medicine, Hong Kong Baptist University, Hong Kong, China; \\ ${ }^{2}$ Research and Development Centre for Natural Health Products, HKBU Shenzhen Research Institute and Continuing Education, \\ Shenzhen, China.
}

\begin{abstract}
Summary
Herba Siegesbeckiae (HS, the dried aerial part of Siegesbeckia orientalis L.) is a commonly used traditional Chinese medicinal herb for treating inflammatory diseases. HS has been reported to exert anti-inflammatory effects by inhibiting the MAPKs and NF-KB pathways, the downstream effectors of TLR4 signalling. This study aims to further investigate the involvement of TLR4 signalling cascades in the effects of an ethanolic extract of HS (HS for short) on inflammatory mediators in murine macrophages. HS was extracted using $\mathbf{5 0 \%}$ ethanol. Lipopolysaccharide (LPS)-stimulated RAW264.7 macrophages were used as the cell model. ELISA was used to detect cytokine/chemokine secretion. Real time-PCR and immunoblotting were used to examine mRNA and protein levels, respectively. We observed that HS dose-dependently inhibited the secretion of PGE 2 , MCP-1, MIP-1 $\alpha$ and RANTES,

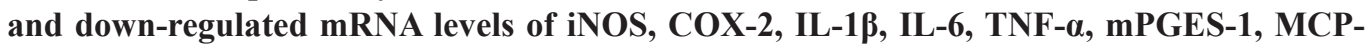
1, MIP-1 $\alpha$ and RANTES in LPS-stimulated RAW264.7 cells. HS did not affect the protein levels of TAK1, TBK1, PI3K, Akt, IKK, c-Jun, c-Fos and IRF3, while, dose-dependently decreased levels of their phosphorylated forms. The protein levels of IRAK1 and IRAK4 were upregulated, while those of TRAF6 and TRAF3 were downregulated by HS. Moreover, the nuclear protein levels of AP-1, NF-KB and IRF3 were dose-dependently decreased by HS. These results indicate that suppression of the IRAK4/MAPKs/AP-1, IRAK4/MAPKs/NF$\kappa B$, IRAK4/PI3K/NF-KB and TRAF3/TBK1/IRF3 pathways is associated with the inhibitory effects of HS on inflammatory mediators in LPS-stimulated RAW264.7 cells. This study provides a pharmacological basis for the clinical application of this herb in the treatment of inflammatory disorders.
\end{abstract}

Keywords: Herba Siegesbeckiae, Siegesbeckia orientalis, Inflammation mediators, AP-1, NF-кB, IRF3, RAW 264.7 macrophages

\section{Introduction}

Herba Siegesbeckiae (HS, Xixiancao in Chinese), as a medicinal herb, was first documented in the Newly Revised Materia Medica (Xinxiu Bencao), issued in

*Address correspondence to:

Dr. Zhiling Yu, School of Chinese Medicine, Hong Kong Baptist University, Kowloon Tong, Hong Kong SAR 999077, China.

E-mail: zlyu@hkbu.edu.hk
A.D. 659 in the Tang Dynasty of China. It functions to eliminate wind-dampness and relieve joint pain $(1,2)$. In the 2015 edition of Chinese Pharmacopeia, it is recorded that the aerial parts of three Siegesbeckia genus plants Siegesbeckia pubescens Makino, S. orientalis L. and S. glabrescens Makino are used as HS (3). In the clinic, HS is widely prescribed to treat inflammatory diseases such as furuncle, arthritis and gout (4). Some preparations made solely from this herb are commercially available (e.g. Xixian Pill/ Tablet) in China and European countries. Of these 
preparations, the patent drug Joint and Muscle Relief Tablets ${ }^{\mathrm{TM}}$ produced by Phynova is the first traditional Chinese medicine licensed under the Traditional Herbal Medicinal Products Directive in the United Kingdom (5). Although there is no contention regarding the use, and efficacy, of HS in the treatment of inflammatory disorders, the mode and mechanism of action of this herb are poorly understood.

Pharmacological studies have demonstrated that HS possesses anti-inflammatory effects in animal models, such as xylene-induced ear edema in mice; cottonball-induced granuloma (6), collagenase-induced paw edema (7) and urate-induced synovitis in rats (4); as well as rheumatoid arthritis induced by mixed arthrogen monoclonal antibody in mice (8). In vitro studies have shown that HS inhibits inflammatory mediators by suppressing the MAPK- and NF- $\kappa B$-pathways in LPSstimulated RAW264.7 cells (9). Kirenol, a natural compound occuring in HS, has been reported to inhibit $\mathrm{NF}-\kappa \mathrm{B}$ activity and exert anti-inflammatory effects in collagen-induced arthritic rats (10). However, these mechanistic studies do not address whether HS/kirenol affect(s) molecular events upstream and/or downstream of NF- $\mathrm{KB}$ and MAPKs, which are downstream effectors of troll-like receptor 4 (TLR4). TLR4 is part of a receptor complex that recognizes and responds to the bacteria lipopolysaccharide (LPS) ligand $(11,12)$. TLR4 signalling pathway activation has been implicated in inflammatory disorders. TLR4 signalling activates transcription factors such as NF-кB, AP-1 and IRF3. These proteins are key inflammatory response mediators (13). In this study we found that inhibition of the IRAK4/MAPKs/AP-

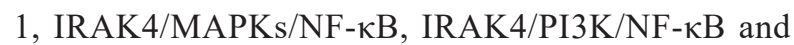
TRAF3/TBK1/IRF3 pathways is associated with the inhibitory effects of HS on inflammatory mediators in LPS-stimulated RAW264.7 cells. These novel findings provide additional justification for the clinical application of HS in the treatment of inflammatory disorders.

\section{Materials and Methods}

\subsection{Reagents and materials}

LPS from Escherichia coli O55:B5, dimethyl sulfoxide (DMSO), 3-(4,5-dimethylthiazol-2-yl)2,5-diphenyltetrazolium bromide (MTT) and Griess reagent were obtained from Sigma Chemicals Ltd. (St. Louis, MO, USA). Penicillin, streptomycin, Dulbecco's Modified Eagle Medium (DMEM) and foetal bovine serum (FBS) were purchased from Hyclone (Logan, UT, USA). Cyclooxygenase-2 (COX-2), Inducible nitric oxide synthase (iNOS), TNF receptor associated factor protein 3 (TRAF3), IKK $\alpha / \beta$, PI3K, phosphoPI3K p85 (Tyr458)/p55 (Tyr199), phospho-IKK $\alpha / \beta$

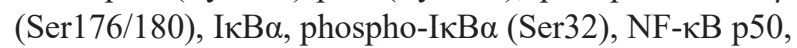
IFN regulatory factor 3 (IRF3), phospho-IRF3 (Ser396), extracellular signal-regulated kinase (ERK), phospho-
ERK (Thr202/Tyr204), c-Jun N-terminal kinase (JNK), phospho-JNK (Thr183/Tyr185), p38 mitogen-activated protein kinase (p38), phospho-p38 (Thr180/Tyr182), TGF $\beta$-activated kinase 1 (TAK1), phospho-TAK 1 (Ser412), interleukin-1 receptor-associated kinase 1 (IRAK1), IRAK4, TANK-binding kinase 1 (TBK1), phospho-TBK1 (Ser172) and GAPDH monoclonal antibodies were obtained from Cell Signalling Technology (Boston, MA, USA). TNF receptor associated factor protein 6 (TRAF6), Akt, phospho-Akt

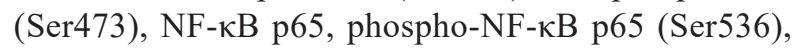
c-Jun, phosphor-c-Jun (Ser63), Pol II (H-224) antibodies were obtained from Santa Cruz Biotechnology (Santa Cruz, CA, USA). Acetonitrile (ACN, HPLC grade) was obtained from RCI Labscan Limited (Thailand). A Milli-Q system (Millipore, MA, USA) was used to prepare ultra-pure water for HPLC analyses. Ethanol (absolute) was from Merck (Darmstadt, Germany). Other materials used in bioassays were from Life Technologies Inc. (GIBICO, USA).

\subsection{Herbal materials}

Three Siegesbeckia genus plants are used as origins of HS in China. However, HS derived from S. orientalis is the most commonly used. In this study, we used the dried aerial part of $S$. orientalis, which originates in Hubei province, China (Longitudes: $108-116^{\circ} \mathrm{E}$, latitudes: $29-33^{\circ} \mathrm{N}$ ). The herb was purchased from the Hubei Shen Nong Traditional Chinese Medicine Co. Ltd and authenticated by Professor Hubiao Chen (Hong Kong Baptist University, HKBU). A voucher specimen (No.20141101) has been deposited at the School of Chinese Medicine, HKBU.

HS is traditionally used in the forms of decoction (extraction using water) and pill made from herb powder, suggesting that the bioactive components of HS may be polar and/or non-polar. Previously, we extracted the herb with three different solvents including water, $50 \%$ ethanol and $95 \%$ ethanol; and compared the inhibitory effect of the three extracts on NO production in LPS-stimulated RAW 264.7 cells. Results indicated that the extract prepared with $50 \%$ ethanol possesses the most potent inhibitory effect on NO production (14). Thus, we used 50\% ethanol to extract the herb in this work. HS (10 g) was grounded and macerated for $1 \mathrm{~h}$ with $100 \mathrm{~mL}$ of $50 \%$ ethanol at room temperature $\left(25 \pm 2{ }^{\circ} \mathrm{C}\right)$, and then extracted by refluxing twice for $1 \mathrm{~h}$ each. The extracts were filtered and combined after cooling; the filtrate and washings were combined and then concentrated by rotary evaporation under reduced pressure to remove the solvent. The concentrated extracts were rapidly frozen at $-80^{\circ} \mathrm{C}$, and then dried in a freeze-dryer (Virtis freeze mobile, Virtis Co., Gardiner, USA). The yield of the extract (HS for short) was $18.03 \%$. To prepare the sample solution for bioassays, HS was freshly dissolved in DMSO, filtered 
with a syringe filter $(0.22 \mu \mathrm{m})$, and then diluted with cell culture medium to various concentrations.

For the quality control of the prepared HS, HPLC analyses were performed on an Agilent 1260 series HPLC-DAD system using a previously described method (15) with some modifications. The separation was conducted on an Alltima ${ }^{\mathrm{TM}} \mathrm{C}-18$ analytical column $(250 \mathrm{~mm} \times 4.6 \mathrm{~mm}$ I.D., $5 \mu \mathrm{m})$ with a gradient mobile phase of solvent A (ultra-pure water) and solvent B (acetonitrile). The HPLC elution profile was as follows: 0-6 $\mathrm{min}, 30 \% \mathrm{~B}$; 6-20 $\mathrm{min}, 30-45 \%$ B; $20-30 \mathrm{~min}, 45 \%$ $\mathrm{B}$. The flow rate was maintained at $1.0 \mathrm{~mL} / \mathrm{min}$ and the column temperature was set at $25^{\circ} \mathrm{C}$. The chromatograms were monitored with the $\mathrm{DAD}$ detector at a wavelength of $215 \mathrm{~nm}$. Each sample of $20 \mu \mathrm{L}$ was injected for analysis.

\subsection{Cell culture}

The RAW267.4 murine macrophage cell line (ATCC TIB-71) was obtained from the American Type Culture Collection (Manassas, VA, USA). The cells were cultured in DMEM at $37^{\circ} \mathrm{C}$ with $5 \% \mathrm{CO}_{2}$ in a humidified incubator. Heat inactivated FBS $(5 \%)$ and penicillin/ streptomycin antibiotic cocktail $(1 \%)$ were included in the culture (16).

\subsection{Cell viability assay}

RAW264.7 cells were seeded in 96-well plates $\left(5 \times 10^{3}\right.$ cells/well) and allowed to adhere overnight. The cells were treated with HS at indicated concentrations for $1 \mathrm{~h}$, and further treated in the presence or absence of LPS (100 ng/mL) for another $24 \mathrm{~h}$. The final concentration of DMSO in the cell culture medium was less than $0.05 \%$ (v/v). Cell viability was evaluated using the MTT assay as described (17).

\subsection{Enzyme-linked immunosorbent assay (ELISA)}

RAW264.7 cells were seeded in 24-well plates $\left(1.5 \times 10^{5}\right.$ cells/well) and allowed to adhere overnight. The cells were treated with indicated concentrations $(\mu \mathrm{g} / \mathrm{mL})$ of HS for $1 \mathrm{~h}$, and then in the presence or absence of LPS $(100 \mathrm{ng} / \mathrm{mL})$ for another $24 \mathrm{~h}$. ELISA kits purchased from eBioscience (San Diego, CA, USA) were used to determine PGE 2 , MCP-1, MIP-1 $\alpha$, IL-1, IL-6, TNF- $\alpha$ and RANTES in the supernatants of the cell cultures following manufacturer's instructions (18).

\subsection{Nitric oxide (NO) production measurement}

The supernatant of cultured RAW 264.7 cells obtained in Section 2.5 was used to measure NO production by determining the accumulated nitrite formed using the Griess reagent according to manufacturer's instructions (Sigma) (19).

\subsection{Real-time polymerase chain reaction analysis}

RAW264.7 cells were seeded in 6 -well plates $(1.0 \times$ $10^{5}$ cells $/ \mathrm{mL}$ ) and allowed for adhere over night. After pretreatment with HS $(80 \mu \mathrm{g} / \mathrm{mL})$ for $1 \mathrm{~h}$, the cells were incubated in the presence or absence of LPS (100 ng/ $\mathrm{mL}$ ) for another $16 \mathrm{~h}$. Trizol reagent (Invitrogen, USA) was used to prepare total RNA. Five $\mu \mathrm{g}$ of RNA was used for reverse transcription by oligo-dT using the SuperScript II Reverse Transcription Kit (Invitrogen, USA). The following primers were used: iNOS (Sense 5'-AGCAACTACTGCTGGTGGTG-3' and anti-sense 5'-TCTTCAGAGTCTGCCCATTG-3'), COX-2 (Sense 5'-CTGGAACATGGACTCACTCAGTTTG-3' and anti-sense 5'-AGGCCTTTGCCACTGCTTGT-3'), IL$1 \beta$ (Sense 5'-GAAGAAGAGCCCATCCTCTG-3' and anti-sense 5'-TCATCTCGGAGCCTGTAGTG-3'), IL-6 (Sense 5'-AGTCCGGAGAGGAGACTTCA-3' and antisense 5'-ATTTCCACGATTTCCCAGAG-3'), TNF- $\alpha$ (Sense 5'-ATGAGAAGTTCCCAAATGGC-3' and antisense 5'-CTCCACTTGGTGGTTTGCTA-3' ), mPGES-1 (Sense 5'-ATGAGGCTGCGGAAGAAGG-3' and antisense 5'-GCCGAGGAAGAGGAAAGGATAG-3'), MCP1 (Sense 5'-AATGCTAACGCCACCGAGAG-3' and antisense 5'-CCTTGTTCTGCTCCTCATAGTCC-3'), MIP$1 \alpha$ (Sense 5'-CCCAGCCAGGTGTCATTTTCC-3' and anti-sense 5'-GCATTCAGTTCCAGGTCAGTG-3'), RANTES (Sense 5'-CATATGGCTCGGACACCA-3' and anti-sense 5'-ACACACTTGGCGGTTCCT-3'), GAPDH (Sense 5'-GGCCTTCCGTGTTCCTACC-3' and antisense 5'-TGCCTGCTTCACCACCTTC-3'); Real-time PCR analysis was conducted using SYBR green reaction mixture in the ABI 7500 Fast Real-time PCR System (Applied Biosystems, USA) (20).

\subsection{Western blotting}

RAW 264.7 cells were seeded in 60-mm-diameter culture dishes $\left(2 \times 10^{5}\right.$ cells $\left./ \mathrm{mL}\right)$ and allowed to adhere overnight. The cells were treated with HS at indicated concentrations for $1 \mathrm{~h}$, and then in the presence or absence of LPS (100 ng/mL) for indicated periods (30, $60 \mathrm{~min}$; or $24 \mathrm{~h}$ ). Cell total extract, cytosolic fraction and nuclear fraction were prepared, respectively. Soluble lysates were immunoblotted with the designated antibodies, and signals were detected by ECL detection reagents (Amersham Biosciences, USA) (21).

\subsection{Statistical analysis}

Data were presented as the mean \pm SD of at least three triplicate determinations. Statistical differences were determined using one-way ANOVA followed by Tukey's multiple comparisons test. GraphPad Prism 5.0 (GraphPad Software, San Diego, CA) was used for statistical analyses. $P<0.05$ was considered to be statistically significant. 


\section{Results and Discussion}

In clinic, HS is commonly prescribed for treating hypertension, malaria, neurasthenia, hepatitis, and inflammatory diseases such as rheumatic arthritis and gout. Phytochemical investigations revealed that this herb contains various types of compounds such as diterpenoids, sesquiterpenoids and flavonoids. Diterpenoids are reported as being major bioactive components of HS (22). Kirenol and darutigenol are two diterpenoids abundant in HS. Kirenol has been shown to inhibit NF- $\kappa \mathrm{B}$ activity and exert anti-inflammatory effects in collagen-induced arthritic rats (10). In this study, the HPLC chromatogram showed that kirenol and darutigenol were present in HS (Figure 1). The mean contents of kirenol and darutigenol in HS were $0.51 \%$ and $0.22 \%$, respectively. Further studies are required to identify the anti-inflammatory compounds in HS.

Previous studies indicated that the MAPKs and $\mathrm{NF}-\kappa \mathrm{B}$ signalling pathways play essential roles in the inhibitory effects of HS on inflammatory mediators $(9,20)$. MAPKs and NF- $\kappa \mathrm{B}$ are components of TLR4 signalling cascades. We believe that inhibition of TLR4 signalling pathways contributes to the inhibitory effects of HS on inflammatory mediators.

In this work, LPS-stimulated RAW264.7 macrophage cells were used as the TLR4-activated cell model to investigate the inhibitory effect of HS on inflammatory mediators. To determine sub-lethal concentrations of HS, MTT assays were first conducted. In the presence or absence of $100 \mathrm{ng} / \mathrm{mL}$ of LPS, the viability of RAW264.7 cells was not significantly altered during a 24-h treatment with up to $120 \mu \mathrm{g} / \mathrm{mL}$ of HS (Figure 2A). HS concentrations of 20,40 and $80 \mu \mathrm{g} / \mathrm{mL}$ were used in subsequent assays.

TLR4 transduces signals through the MyD88dependent and MyD88-independent pathways, which are mainly mediated by the activation of transcription factors NF- $\mathrm{B}, \mathrm{AP}-1$ and IRF3. Upon LPS stimulation, $\mathrm{NF}-\kappa \mathrm{B}$ and $\mathrm{AP}-1$ are activated via the MyD88dependent pathway, and IRF3 is activated via the MyD88-independent pathway in macrophages (13). We have determined the effect of HS on pro-inflammatory mediators regulated by the three transcription factors in LPS-induced RAW264.7 cells, and observed that HS concentration-dependently reduced the production of $\mathrm{PGE}_{2}$ (regulated by NF- $\kappa \mathrm{B}$ ), MCP-1, MIP-1 $\alpha$ (regulated by AP-1), and RANTES (regulated by IRF3) (Figure 2B), and lowered mRNA levels of iNOS, COX-2, IL$1 \beta$, IL-6, TNF- $\alpha$, mPGES-1, MCP-1, MIP- $1 \alpha$ and RANTES (Figure 2C). Additionally, HS inhibited other NF- $\kappa$ B-regulated inflammatory mediators that have previously been reported $(9,20)$. These mediators have been examined (Supplementary Figure 1S, http://www. biosciencetrends.com/action/getSupplementalData. php? ID =26) and discussed in the subsequent sections.

We determined the effect of HS on the nuclear localization of transcription factors NF- $\kappa \mathrm{B}, \mathrm{AP}-1$ and IRF3 in LPS-induced RAW264.7 cells. Our results indicate that the nuclear protein levels of NF$\kappa \mathrm{B}$ subunits (p65 and p50), AP-1 subunits (c-Jun and c-Fos) and IRF3 were markedly increased upon LPS stimulation. HS concentration-dependently reversed the increment (Figure 3A). Moreover, LPS-induced downregulation of p65, p50, c-Jun and c-Fos in the cytoplasm was dose-dependently inhibited by HS (Figure 3B). Cytoplasmic IRF3 levels were not significantly altered by the treatments (data not shown). These results demonstrate that HS inhibits the production of proinflammatory mediators regulated by NF- $\mathrm{B}$, AP-1 and IRF3, suggesting that both MyD88-dependent and MyD88-independent pathways are involved in the antiinflammatory effects of HS.

Upon activation of MyD88-dependent signalling, MyD88 recruits IRAK4, thereby allowing the association of IRAK1. The TLR4 receptor complex-associated IRAK1 is phosphorylated by IRAK4, which in turn promotes the autophosphorylation and dissociation of IRAK1 from the receptor complex. Subsequently, IRAK1 interacts with TAK1-TAB1-TAB2 kinase complex-associated TRAF6. This interaction leads to the ubiquitylation of TRAF6, which induces the activation of TAK1 (23). The activated TAK1 induces the phosphorylation of MAPKs and IKK, leading to the activation of transcription factors NF-kB and AP-1 $(24,25)$. Upon activation of the MyD88-independent pathway, the TRAF3/TBK1 complex is activated, resulting in the phosphorylation and nuclear localization of IRF3 (26). Our results showed that HS inhibited LPS-induced degradation of IRAK 1 and IRAK4, concentration-dependently lowered protein levels of TRAF6, TRAF3, and phosphorylated forms of TAK1 and TBK1 (Figure 4A). These data further suggest the involvement of both MyD88-dependent and MyD88independent pathways in the effects of HS.

Activation of IKK kinase initiates the phosphorylation and degradation of $\mathrm{I} \kappa \mathrm{B} \alpha$ resulting in the release of p50-p65 for nuclear translocation, and the eventual mediation of NF- $\kappa \mathrm{B}$-dependent transcriptional activity (27). Studies have demonstrated that the $\mathrm{PI} 3 \mathrm{~K} / \mathrm{Akt}$ pathway can regulate $\mathrm{NF}-\kappa \mathrm{B}$ signalling positively or negatively $(28,29)$. Our results showed that HS concentration-dependently inhibited the phosphorylation of MAPKs (JNK, ERK and p38), IкB $\alpha$ and NF- $\kappa \mathrm{B}$ p65. HS also inhibited the protein expression of iNOS and COX-2, the secretion of cytokines (IL$1 \beta$, IL- 6 and TNF- $\alpha$ ) and the production of NO in a concentration-dependent manner (Supplementary Figure 1S, http://www.biosciencetrends.com/action/ getSupplementalData.php?ID=26). These results are consistent with observations in previous reports $(9,20)$ and thus confirm the involevement of MAPKs and $\mathrm{NF}-\kappa \mathrm{B}$ signalling in the effects of HS. Our results also showed that HS concentration-dependently inhibited 


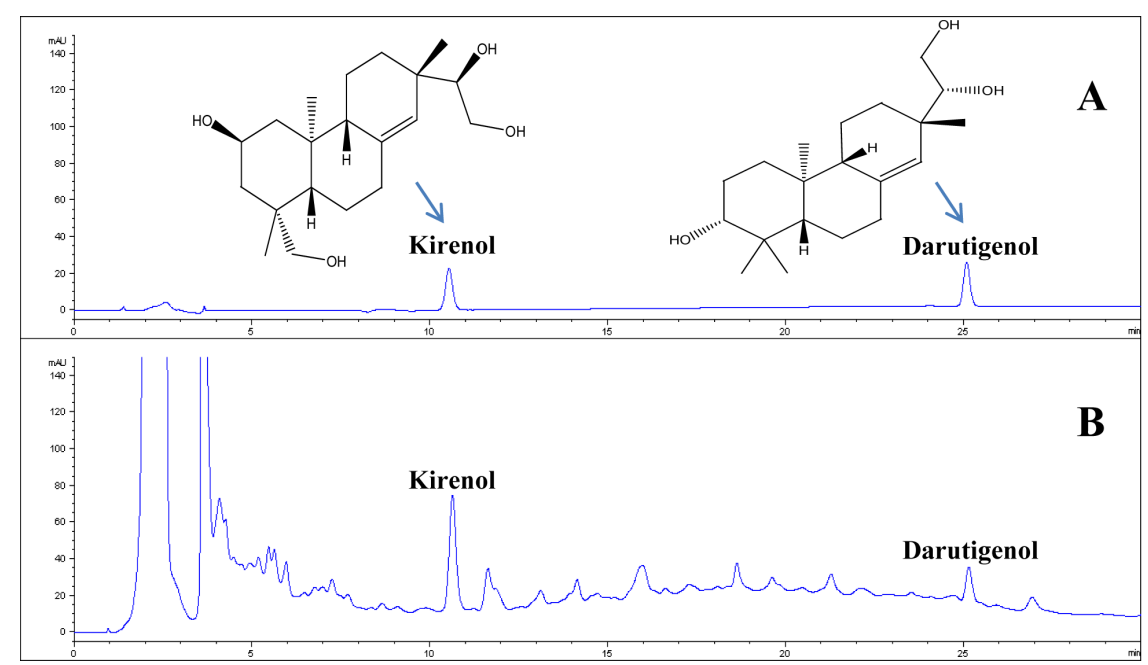

Figure 1. HPLC analyses of HS and mixed standards (kirenol and darutigennol) (A. Mixed standards; B. HS sample).

A
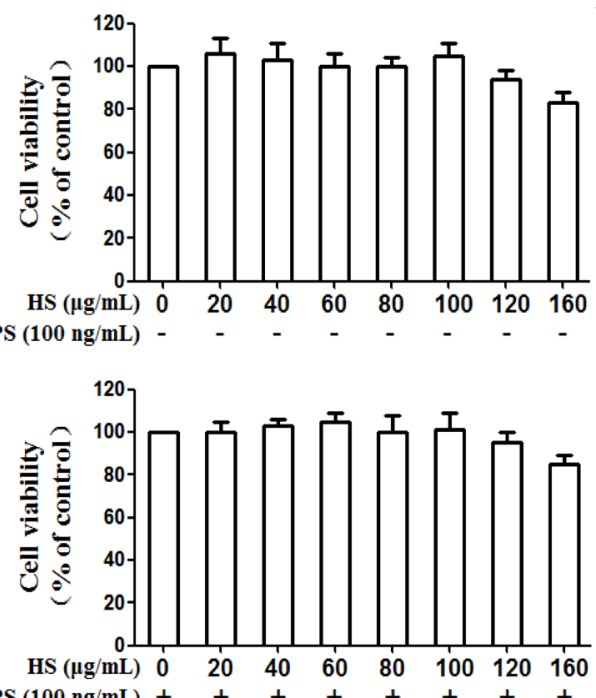

B
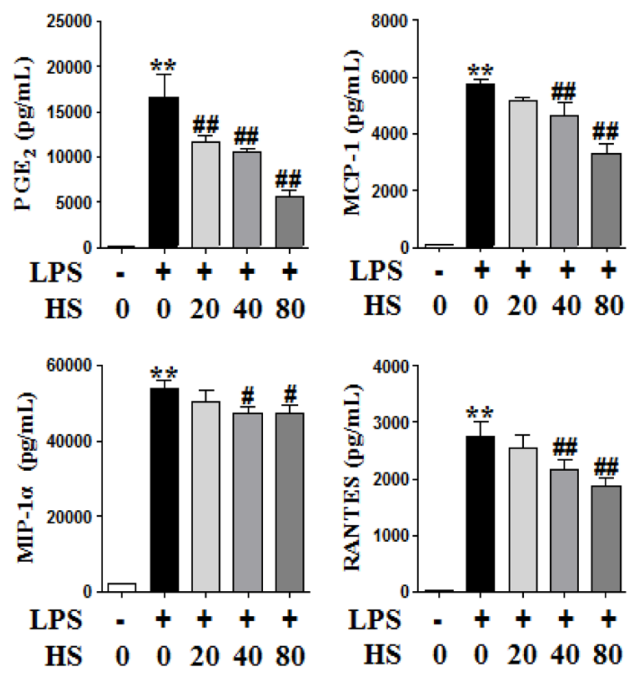

C
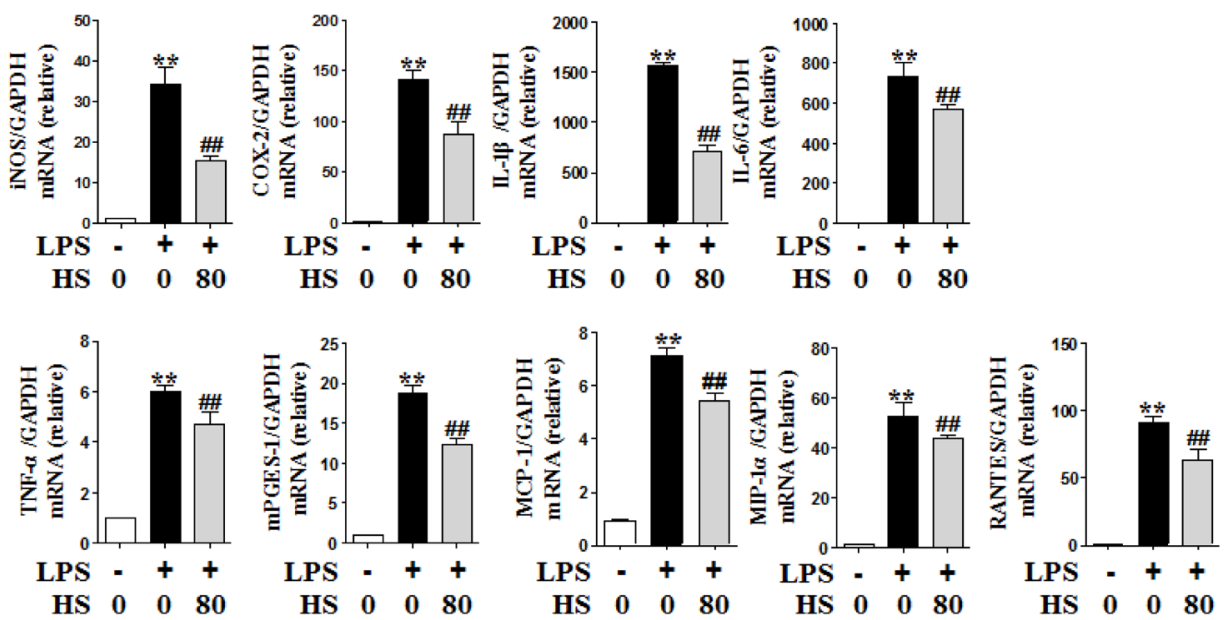

Figure 2. HS inhibited inflammatory mediators in LPS-stimulated RAW 264.7 cells. (A) Effects of HS on the cell viability in the absence (upper panel) or presence of LPS (lower panel). (B) HS reduced the production of PGE, MCP-1, MIP-1 $\alpha$ and RANTES. (C) HS decreased mRNA levels of iNOS, COX-2, IL-1 $\beta$, IL-6, TNF- $\alpha$, mPGES-1, MCP-1, MIP-1 $\alpha$ and RANTES in LPS-stimulated RAW 264.7 cells. Data are shown as mean \pm SD from tree independent experiments. ${ }^{* *} P<0.01$ vs. value in corresponding control; ${ }^{\#} P<0.01, \# P<0.05$ vs. value in cells treated with LPS alone. 
A

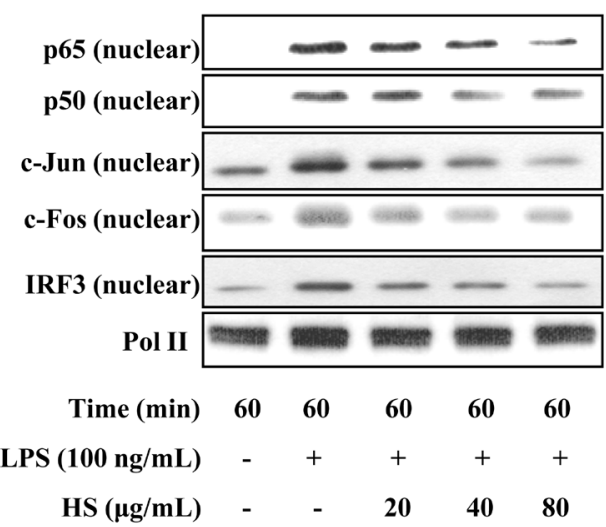

B

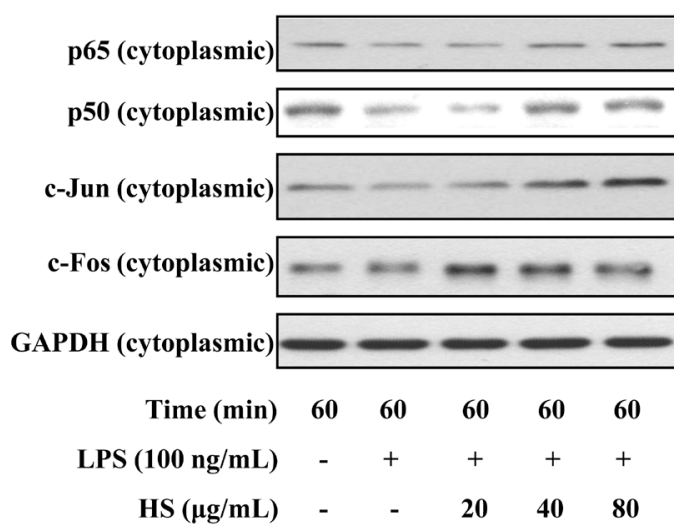

Figure 3. Effects of HS on the nuclear localization of the three transcription factors in LPS-induced RAW264.7 cells. (A) $\mathrm{HS}$ decreased nuclear protein levels of NF-kB subunits (p65 and p50), AP-1 subunits (c-Jun and c-Fos) and IRF3. (B) HS inhibited down-regulation of cytoplasmic protein levels of p65, p50, c-Jun and c-Fos. Results of a typical experiment from three independent ones are shown.

A

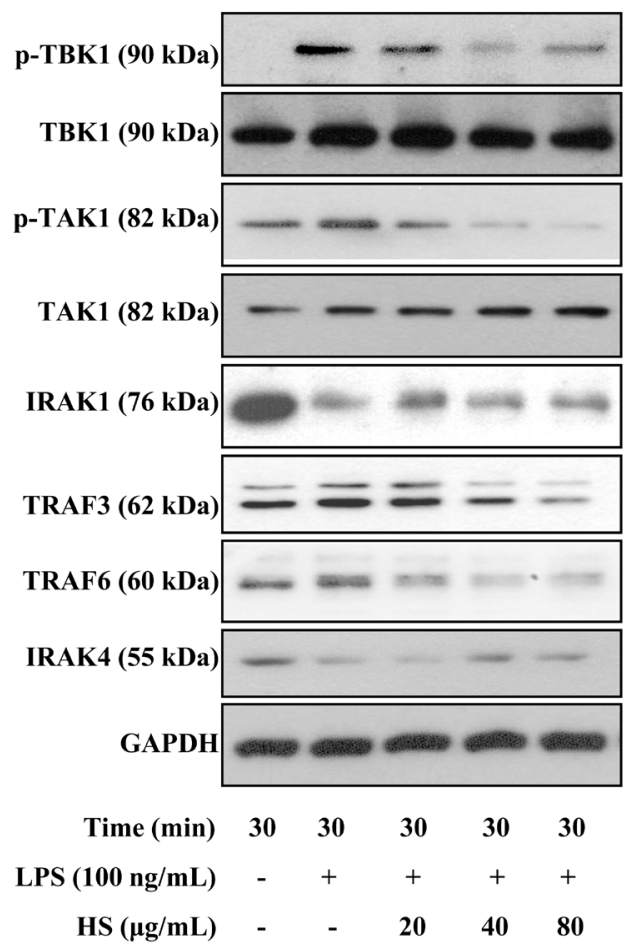

B

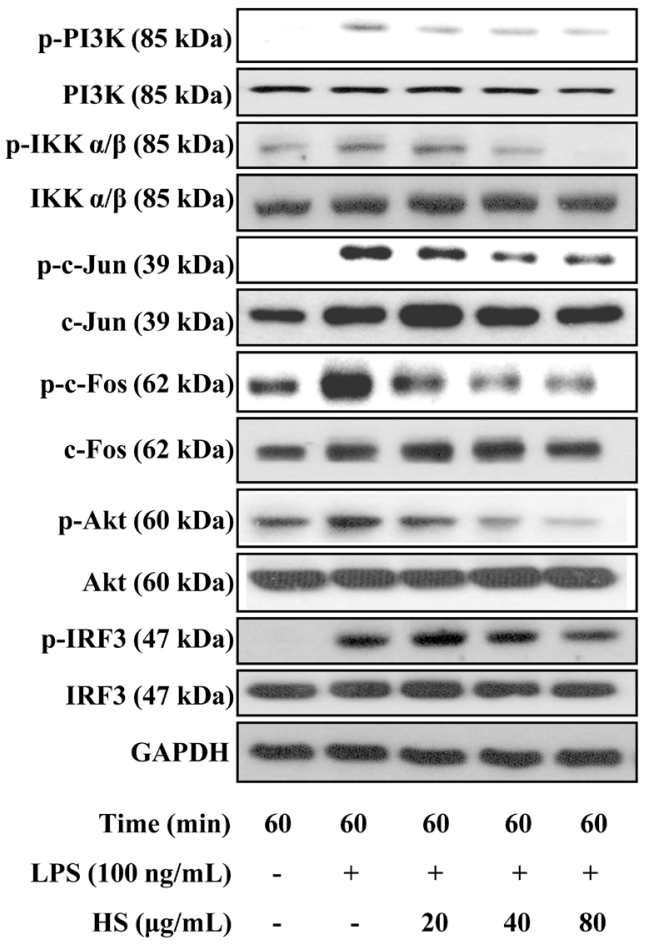

Figure 4. Effects of HS on molecules involved in the TLR4 signaling pathways in LPS-stimulated RAW 264.7 cells. (A) Effects of HS on protein levels of TRAF6, TRAF3, IRAK1, IRAK4, phospho-TAK1, TAK1, phospho-TBK1 and TBK1. (B) Effects of HS on protein levels of phosphorylated and nonphosphorylated forms of PI3K, Akt, IKK, c-Jun, c-Fos and IRF3. Results of a typical experiment from three independent ones are shown.

LPS-induced phosphorylation of PI3K, Akt, IKK, c-Jun, c-Fos and IRF3 (Figure 4B). These findings support the involvement of the MyD88-dependent pathways of MAPKs/AP-1 and PI3K/Akt, and the MyD88independent pathway TBK1/IRF3 in the effects of HS. As stated previously, the role of Akt signalling in NF$\kappa \mathrm{B}$ activation is still controversial. Further studies are needed to delineate the inhibitory effect of HS on the Akt/NF-kB pathway.
Although we examined the proteins levels of TLR4, MyD88 and TRAM (data not shown) following HS treatment, we did not observe any significant changes. Previous studies suggest that some agents can alter the interaction between TLR4 receptor complex and LPS by competitively binding to the extracellular domain of TLR4 (30). Therefore, further studies are needed to determine the possible abrogation of LPS-TLR4 interaction by HS in RAW264.7 cells. 


\section{Conclusion}

In this study, our results indicate that inhibition of the IRAK4/MAPKs/AP-1, IRAK4/MAPKs/NF- $\kappa$ B, IRAK4/PI3K/NF- $\mathrm{BB}$ and TRAF3/TBK1/IRF3 pathways is associated with the inhibitory effects of HS on inflammatory mediators in LPS-stimulated RAW264.7 cells. Therefore, not only do our experiments support the use of HS as a medicinal herb in the treatment of various inflammatory conditions, they also provide a novel pharmacological and mechanistic insight into the fundamentals of its activity. However, additional studies are warranted to further explore the molecular mechanism of action and identify the bioactive components of HS.

\section{Acknowledgements}

This study was supported by the Research Grants Council of Hong Kong (GRF12125116); National Natural Science Foundation of China (81673649); the Food and Health Bureau (HMRF14150571), Natural Science Foundation of Guangdong Province (2016A030313007); Science, Technology and Innovation Commission of Shenzhen (JCYJ20150630164505508, JCYJ20160229210327924 and JCYJ20170817173608483) and Hong Kong Baptist University (FRG1/16-17/048 and FRG2/17-18/032).

\section{References}

1. Wang JP, Zhou YM, Ye YJ, Shang XM, Cai YL, Xiong $\mathrm{CM}, \mathrm{Wu} Y X, \mathrm{Xu} \mathrm{HX}$. Topical anti-inflammatory and analgesic activity of kirenol isolated from Siegesbeckia orientalis. J Ethnopharmacol. 2011; 137:1089-1094.

2. Wang LL, Hu LH. Chemical constituents of Siegesbeckia orientalis L. J Integr Plant Biol. 2006; 48:991-995.

3. The State Pharmacopoeia Commission of PR China. Chinese Pharmacopoeia. China Medical Science and Technology Press, Beijing, China, 2015; Part I:pp.368. (in Chinese)

4. Nguyen TD, Thuong PT, Hwang IH, Hoang TK, Nguyen MK, Nguyen HA, Na M. Anti-Hyperuricemic, AntiInflammatory and Analgesic Effects of Siegesbeckia orientalis L. Resulting from the Fraction with High Phenolic Content. BMC Complement Altern Med. 2017; 17:191.

5. Phynova. Phynova Joint and Muscle Relief Tablets ${ }^{\mathrm{TM}}$. https://www.phynova.com/products/phynova-joint-andmuscle-relief-tablets/ (accessd March 01, 2018).

6. Liu DY, Hu HH. Experimental Research on antiinflammation of processed Herba Siegesbeckiae. Lishenzhen medicine and materia medica research. 2008; 19:668-669.

7. Huh JE, Baek YH, Lee JD, Choi DY, Park DS. Therapeutic effect of Siegesbeckia pubescens on cartilage protection in a rabbit collagenase-induced model of osteoarthritis. J Pharmacol Sci. 2008; 107:317-328.

8. Fu XC, Jiang FP, Fan JZ, Bai HB. Extract of Herba Siegesbeckiae on mouse rheumatoid arthritis induced by anthrogen-CIA monoclonal antibody. Zhejiang Da Xue
Xue Bao Yi Xue Ban. 2013; 42:556-560. (in Chinese)

9. Hong YH, Weng LW, Chang CC, Hsu HF, Wang CP, Wang SW, Houng JY. Anti-inflammatory effects of Siegesbeckia orientalis ethanol extract in in vitro and in vivo models. Biomed Res Int. 2014; 2014:329712.

10. Wang ZM, Zhu SG, Wu ZW, Lu Y, Fu HZ, Qian RQ. Kirenol upregulates nuclear annexin-1 which interacts with NF-kappaB to attenuate synovial inflammation of collagen-induced arthritis in rats. J Ethnopharmacol. 2011; 137:774-782.

11. Aminlari A, Richard KD, Zhang HY, O'Donnell DP, Wang GS, Alarcon WH, Steinstraesser L, Wang SC, Remick DG, $\mathrm{Su}$ GL. LPS activation of kupffer cells is mediated by toll like receptor 4 (TLR 4). Hepatology. 1999; 30:374a-374a.

12. Panzer S. Differential response to LPS isotypes induced platelet activation mediated by Toll-like receptor (TLR)-4. Clin Immunol. 2013; 146:13-14.

13. Lu YC, Yeh WC, Ohashi PS. LPS/TLR4 signal transduction pathway. Cytokine. 2008; 42:145-151.

14. Guo H, Zhang Y, Cheng BC, Lau MY, Fu XQ, Li T, Su T, Zhu PL, Chan YC, Tse AK, Yi T, Chen HB, Yu ZL. Comparison of the chemical profiles and inflammatory mediator-inhibitory effects of three Siegesbeckia herbs used as Herba Siegesbeckiae (Xixiancao). BMC Complement Altern Med. 2018; 18:141.

15. Yan DX, Wang YJ, Duan Q, Zhang XM, Zhao HQ. Simultaneous Determination of Kirenol and Darutigenol in Herba Siegesbeckiae by RP-HPLC. Chinese Pharmaceutical Journal. 2010; 45:945-948. (in Chinese)

16. Su T, Bai JX, Chen YJ, Wang XN, Fu XQ, Li T, Guo H, Zhu PL, Wang Y, Yu ZL. An Ethanolic Extract of Ampelopsis Radix Exerts Anti-colorectal Cancer Effects and Potently Inhibits STAT3 Signaling In Vitro. Front Pharmacol. 2017; 8:227.

17. Gerlier D, Thomasset N. Use of Mtt Colorimetric Assay to Measure Cell Activation. J Immunol Methods. 1986; 94:57-63.

18. Du J, Cheng BCY, Fu XQ, Su T, Li T, Guo H, Li SM, Wu JF, Yu H, Huang WH, Cao H, Yu ZL. In vitro assays suggest Shenqi Fuzheng Injection has the potential to alter melanoma immune microenvironment. J Ethnopharmacol. 2016; 194:15-19.

19. Green LC, Wagner DA, Glogowski J, Skipper PL, Wishnok JS, Tannenbaum SR. Analysis of nitrate, nitrite, and $\left[{ }^{15} \mathrm{~N}\right]$ nitrate in biological fluids. Anal Biochem. 1982; 126:131-138.

20. Su T, Yu H, Kwan HY, Ma XQ, Cao HH, Cheng CY, Leung AK, Chan CL, Li WD, Cao H, Fong WF, Yu ZL. Comparisons of the chemical profiles, cytotoxicities and anti-inflammatory effects of raw and rice wine-processed Herba Siegesbeckiae. J Ethnopharmacol. 2014; 156:365369.

21. Cheng BC, Yu H, Su T, Fu XQ, Guo H, Li T, Cao HH, Tse AK, Kwan HY, Yu ZL. A herbal formula comprising Rosae Multiflorae Fructus and Lonicerae Japonicae Flos inhibits the production of inflammatory mediators and the IRAK-1/TAK1 and TBK1/IRF3 pathways in RAW 264.7 and THP-1 cells. J Ethnopharmacol. 2015; 174:195-199.

22. Huo L, Jiang Z, Li H, Wang M, Ye X, Ji B, Guo X. Simultaneous determination of seven major diterpenoids in Siegesbeckia pubescens Makino by high-performance liquid chromatography coupled with evaporative light scattering detection. J Sep Sci. 2012; 35:2585-2591.

23. Akira S, Takeda K. Toll-like receptor signalling. Nat Rev Immunol. 2004; 4:499-511. 
24. Hipskind RA, Bilbe G. MAP kinase signaling cascades and gene expression in osteoblasts. Front Biosci. 1998; $3: \mathrm{d} 804-816$.

25. Schulze-Osthoff K, Ferrari D, Riehemann K, Wesselborg S. Regulation of NF- $\kappa$ B Activation by MAP Kinase Cascades. Immunobiology. 1997; 198:35-49.

26. Kim SS, Lee KG, Chin CS, Ng SK, Pereira NA, Xu S, Lam KP. DOK3 is required for IFN-beta production by enabling TRAF3/TBK1 complex formation and IRF3 activation. J Immunol. 2014; 193:840-848.

27. Cianciulli A, Calvello R, Porro C, Trotta T, Salvatore R, Panaro MA. PI3k/Akt signalling pathway plays a crucial role in the anti-inflammatory effects of curcumin in LPSactivated microglia. Int Immunopharmacol. 2016; 36:282290.

28. Bauerfeld CP, Rastogi R, Pirockinaite G, Lee I, Huttemann
M, Monks B, Birnbaum MJ, Franchi L, Nunez G, Samavati L. TLR4-Mediated AKT Activation Is MyD88/ TRIF Dependent and Critical for Induction of Oxidative Phosphorylation and Mitochondrial Transcription Factor A in Murine Macrophages. J Immunol. 2012; 188:28472857.

29. Luyendyk JP, Schabbauer GA, Tencati M, Holscher T, Pawlinski R, Mackman N. Genetic analysis of the role of the PI3K-Akt pathway in lipopolysaccharide-induced cytokine and tissue factor gene expression in monocytes/ macrophages. J Immunol. 2008; 180:4218-4226.

30. Brodsky I, Medzhitov R. Two modes of ligand recognition by TLRs. Cell. 2007; 130:979-981.

(Received May 15, 2018; Revised June 23, 2018; Accepted June 26, 2018) 